\title{
Unsupervised Calculation of Free Energy Barriers in Large Crystalline Systems
}

\author{
Thomas D. Swinburne \\ CCFE, Culham Science Centre, Abingdon, Oxon OX14 3DB, United Kingdom \\ Mihai-Cosmin Marinica \\ DEN-Service de Recherches de Métallurgie Physique, CEA, Université Paris-Saclay, F-91191 Gif-sur-Yvette, France
}

(Received 15 July 2017; published 29 March 2018)

\begin{abstract}
The calculation of free energy differences for thermally activated mechanisms in the solid state are routinely hindered by the inability to define a set of collective variable functions that accurately describe the mechanism under study. Even when possible, the requirement of descriptors for each mechanism under study prevents implementation of free energy calculations in the growing range of automated material simulation schemes. We provide a solution, deriving a path-based, exact expression for free energy differences in the solid state which does not require a converged reaction pathway, collective variable functions, Gram matrix evaluations, or probability flux-based estimators. The generality and efficiency of our method is demonstrated on a complex transformation of $C 15$ interstitial defects in iron and double kink nucleation on a screw dislocation in tungsten, the latter system consisting of more than 120000 atoms. Both cases exhibit significant anharmonicity under experimentally relevant temperatures.
\end{abstract}

DOI: 10.1103/PhysRevLett.120.135503

Thermally activated mechanisms play a crucial role in all materials science phenomena, e.g., body-centered cubic (bcc) plasticity [1], creep [2], postirradiation annealing [3], and numerous others [4]. Thermally activated mechanisms are in large part described by the free energy profile $\mathcal{F}(\xi)$ defined with respect to some reaction coordinate $\xi \in[0,1]$ between the initial and final states [5]. As is well known, the free energy barrier $\Delta \mathcal{F} \equiv \max [\mathcal{F}(\xi)]-\min [\mathcal{F}(\xi)]$ is widely used in the transition state theory [6] rate $k=\omega_{0} \exp (-\beta \Delta \mathcal{F})$, while the total free energy difference $\mathcal{F}(1)-\mathcal{F}(0)$ gives the ratio of equilibrium populations $n_{1} / n_{0}=\exp \{-\beta[\mathcal{F}(1)-\mathcal{F}(0)]\}$. Both quantities require accurate calculation of $\mathcal{F}(\xi)$ and are of critical importance to materials simulation $[4,7,8]$. However, materials science applications are typically forced to use approximate harmonic methods in the absence of any systematic tool to probe anharmonicity, an issue which this Letter aims to address.

The free energy profile is defined as a conditional average through [9]

$\mathcal{F}(\xi)=-\beta^{-1} \ln \left|\int d \mathbf{X} \exp (-\beta V(\mathbf{X})) \delta(\xi-\tilde{\xi}(X))\right|$,

where $V(\mathbf{X})$ is the system potential energy and $\tilde{\xi}: \mathbb{R}^{3 N} \rightarrow$ $[0,1]$ takes a given set of $N$ atomic positions $\mathbf{X} \in \mathbb{R}^{3 N}$ and returns a reaction coordinate $\xi \in[0,1]$. Determining a suitable $\tilde{\xi}$ is a crucial component of all free energy calculation techniques and is a central topic of this Letter.

A powerful array of methods have been developed to calculate $\mathcal{F}(\xi)$ when $\xi$ can be determined through one or more collective variable functions which accurately describe the transformation under study. The collective variable function(s) either return $\xi$ directly [10-13] or a reaction pathway is found in the collective variable space [14-18], with $\xi$ defined as an affine parameter along the reaction path. Collective variable-based methods have found enormous successes in studies of molecular systems as the reduced dimensionality permits rapid exploration while accommodating the typically very large entropic effects [14]. However, the application of these methods to materials science problems such as dislocation migration [1] or point defect cluster transformations [19] is hindered by the general inability to define a suitable set of collective variable functions outside of a few simple cases [20]. This is well recognized as a critical problem for the implementation of free energy methods to go beyond harmonic approximation in automated, unsupervised, simulation schemes such as adaptive kinetic Monte Carlo calculations [8,21-23], accelerated molecular dynamics [24,25], and a rapidly growing number of statistical learning approaches [26-28] that represent an active forefront of materials simulation.

In this Letter, we detail a new path-based free energy calculation technique, which permits calculation of anharmonic free energy profiles for complex materials science problems without the requirement to define any collective variable functions or converge a true minimum free energy pathway. Our method allows a temperature dependent pathway and fully anharmonic thermal vibrations with a $O(N)$ computational demand and ideal parallel scalability in the ensemble average sampling [9]. We only require a reference pathway which is suitably local to the true path, here chosen initially to be the minimum energy path 
(MEP), which is ideally suited to automated, unsupervised implementation in a wide class of simulation schemes. A new expression for the free energy gradient is derived that defines $\xi$ with respect to the MEP but nevertheless is valid for a temperature dependent reaction pathway under locality conditions appropriate for solid state systems. Our approach provides a clear signature of when this locality condition is violated, and an iterative scheme is described to accommodate such cases. Our main result also greatly simplifies a Jacobian term that has plagued previous path-based techniques $[9,29,30]$.

A popular path-based approach is the finite temperature string method [16,31-33], which is typically applied in collective variable space though in some notable cases has been applied in the full configuration space $[34,35]$. The method converges a discretized reaction pathway at finite temperature through the use of reflective Voronoi cells around each discretization point, before calculating the change in free energy across each cell through a population flux-based estimator. This is a powerful and successful method for finding free energy paths in molecular systems which have a poorly defined reference structure at finite temperature; however, to accommodate these large fluctuations, the user must determine an application specific parametrization, including a smoothing parameter for the reaction pathway which may obscure important features of the free energy landscape in materials systems. By using the MEP, we can accurately compute pathway tangents that are very challenging to obtain from time averaged structures, offering significant advantages for automated application in solid state systems.

We demonstrate our method on two examples of great practical importance in materials science-for which tens to thousands of atoms participate in the diffusion process and are impossible to address using standard free energy calculation techniques - a complex transformation of interstitial clusters in iron and the migration of $\langle 111\rangle$ screw dislocations in tungsten, the latter example requiring a system size of around 120000 atoms. In both cases, our method does not require any ad hoc parametrization, and because of the $O(N)$ efficiency, the full anharmonic free energy can be evaluated for an equivalent cost to an $O(N)$ harmonic approximation [36], significantly improving on the standard $O\left(N^{3}\right)$ implementation.

Method.-The central goal of our method is to evaluate the free energy gradient (or mean force) $\partial_{\xi} \mathcal{F}(\xi)$, which can be integrated to give the free energy difference $\mathcal{F}(\xi)-\mathcal{F}(0)=\int_{0}^{\xi} \partial_{\xi} \mathcal{F}\left(\xi^{\prime}\right) d \xi^{\prime}$. We treat a system of $N$ atoms with a vector of atomic positions $\mathbf{X} \in \mathbb{R}^{3 N}$ subject to a gradient force $-\boldsymbol{\nabla} V(\mathbf{X})$. Having identified the desired initial and final states, a nudged elastic band (NEB) [37] calculation produces an MEP $\mathbf{X}_{0}(\xi)$ at a discrete set of points $\left\{\xi_{i}\right\}$, with intermediate configurations produced through spline interpolation for each atomic coordinate. By taking the derivative of the spline interpolation, we can also introduce the pathway tangent vector $\partial_{\xi} \mathbf{X}_{0}(\xi)$. With these quantities, we can now construct a reaction coordinate function $\tilde{\xi}(\mathbf{X})$, defined to return the value of $\xi$ which corresponds to the closest point on $\mathbf{X}_{0}(\xi)$ to $\mathbf{X}$.

By minimizing the distance $\sqrt{\left|\mathbf{X}-\mathbf{X}_{0}(\xi)\right|^{2}}$ with respect to $\xi$, the reaction coordinate $\xi$ satisfies the zero derivative condition

$$
\partial_{\xi} \mathbf{X}_{0}(\xi) \cdot\left[\mathbf{X}-\mathbf{X}_{0}(\xi)\right]=0 .
$$

Equation (2) defines a subspace of configurations lying on a $3 N-1$ dimensional hyperplane normal to the pathway tangent $\partial_{\xi} \mathbf{X}_{0}(\xi)$. At a finite temperature, the reaction pathway may change. However, providing the new finite temperature pathway remains in a "tube" surrounding the MEP, and this definition of the reaction coordinate remains valid at a finite temperature. This locality condition is common with all path-based methods [16]; though in the following, we derive a more precise formulation of this condition and a general strategy to account for very large pathway deviations.

Under this locality condition, the function $\tilde{\xi}(\mathbf{X})$, defined implicitly as the solution of (2), can now be used in the formally exact free energy gradient at a finite temperature $T[12]$

$$
\partial_{\xi} \mathcal{F}(\xi ; T)=\left\langle\frac{\mathbf{w} \cdot \boldsymbol{\nabla} V}{\mathbf{w} \cdot \boldsymbol{\nabla} \tilde{\xi}}+\beta^{-1} \boldsymbol{\nabla} \cdot \frac{\mathbf{w}}{\mathbf{w} \cdot \boldsymbol{\nabla} \tilde{\xi}}\right\rangle_{\xi},
$$

where the average is over all configurations satisfying (2) such that $\tilde{\xi}(\mathbf{X})=\xi$ and $\mathbf{w}$ is an arbitrary vector function [38] that satisfies $\mathbf{w} \cdot \boldsymbol{\nabla} \tilde{\xi}>0$, which here is set to the zero temperature pathway tangent, i.e., $\mathbf{w}=\partial_{\xi} \mathbf{X}_{0}$. The condition $\partial_{\xi} \mathbf{X}_{0} \cdot \nabla \tilde{\xi}>0$ is a mathematical restatement of the locality condition, namely, that the finite temperature pathway tangent has a positive projection along the MEP tangent $[9,18]$.

The second term in (3) derives from the Jacobian of the nonlinear transformation that maps $\mathbf{X} \rightarrow \xi$ and requires the $O\left(N^{2}\right)$ evaluation of the $3 N \times 3 N$ Gram matrix $\boldsymbol{\nabla} \otimes \boldsymbol{\nabla} \tilde{\xi}$. Evaluating this matrix at each time step has been a critical issue in applying constrained path-based methods to large systems [9].

A tractable form for $\partial_{\xi} \mathcal{F}$ can be derived by first noting that $\boldsymbol{\nabla} \tilde{\xi}$ must be proportional to $\partial_{\xi} \mathbf{X}_{0}$, as all displacements perpendicular to $\partial_{\xi} \mathbf{X}_{0}$ leave $\xi$ unchanged by definition in Eq. (2). By considering small displacements $\delta \mathbf{X}$ and $\delta \xi$ which maintain (2), we obtain

$$
\boldsymbol{\nabla} \tilde{\xi}=\frac{\partial_{\xi} \mathbf{X}_{0}}{\psi(\mathbf{X}, \xi)\left|\partial_{\xi} \mathbf{X}_{0}\right|^{2}},
$$

where $|\cdots|$ is the Euclidean norm, and the scalar function $\psi(\mathbf{X}, \xi)$ writes as 


$$
\psi(\mathbf{X}, \xi)=\left(1-\frac{\partial_{\xi}^{2} \mathbf{X}_{0}}{\left|\partial_{\xi} \mathbf{X}_{0}\right|^{2}} \cdot\left[\mathbf{X}-\mathbf{X}_{0}\right]\right)
$$

Crucially, this expression for $\boldsymbol{\nabla} \tilde{\xi}$ only requires derivatives of the MEP $\mathbf{X}_{0}(\xi)$ with respect to $\xi$, an $O(N)$ operation which can be calculated once, to arbitrary accuracy, before any simulation run. The locality condition $\partial_{\xi} \mathbf{X}_{0} \cdot \nabla \tilde{\xi}>0$ is now equivalent to

$$
\psi(\mathbf{X}, \xi)>0 .
$$

Through use of (2), one finds

$$
\langle\psi\rangle_{\xi}=\frac{\partial_{\xi} \mathbf{X}_{\mathrm{T}} \cdot \partial_{\xi} \mathbf{X}_{0}}{\left|\partial_{\xi} \mathbf{X}_{0}\right|^{2}},
$$

where $\mathbf{X}_{\mathrm{T}}(\xi)=\langle\mathbf{X} ; \mathrm{T}\rangle_{\xi}$ is the finite temperature minimum free energy path (MFEP), thus demonstrating that the locality condition (6) requires that the MFEP tangent has a positive projection on the MEP tangent. Under conditions where (6) is satisfied, the exact free energy gradient takes the simple form

$$
\partial_{\xi} \mathcal{F}(\xi)=\left\langle\psi(\mathbf{X}, \xi) \partial_{\xi} \mathbf{X}_{0} \cdot \nabla V+\beta^{-1} \partial_{\xi} \ln \frac{|\psi(\mathbf{X}, \xi)|}{\left|\partial_{\xi} \mathbf{X}_{0}\right|}\right\rangle_{\xi} .
$$

Equation (8) is our main result, an expression for the free energy gradient along a finite temperature reaction pathway $\mathbf{X}_{\mathrm{T}}(\xi)$ using only some candidate pathway [here, the MEP $\mathbf{X}_{0}(\xi)$ ], under the locality assumption (6). A key advantage is that, irrespective of the candidate pathway, the free energy landscape will be strictly parabolic around the MFEP, where it can be shown [9] that sampling with overdamped Langevin dynamics is optimal. The finite temperature pathway is still calculated in our simulations, as is $\langle\psi\rangle_{\xi}$, allowing us to verify the validity of our key approximation (6), which should be checked systematically for the system of interest. We have found (6) to be satisfied for all cases presented below, even when the pathway undergoes nontrivial changes at finite temperature, as can be seen in Fig. 2.

If at some high temperature $T_{H}$, the locality condition $\psi(\mathbf{X}, \xi)>0$ when using the MEP is regularly violated, and a new candidate pathway $\tilde{\mathbf{X}}_{0}(\xi)$ should be used instead of the MEP such that (8) is satisfied with $\mathbf{X}_{0}(\xi) \rightarrow \tilde{\mathbf{X}}_{0}(\xi)$. Crucially, while the new candidate path $\tilde{\mathbf{X}}_{0}(\xi)$ should have better locality to the MFEP, we do not have to produce a converged calculation of the MFEP $\mathbf{X}_{T_{H}}(\xi)$. This provides a powerful strategy to avoid the well-known numerical difficulties in evaluating tangents of $\mathbf{X}_{T_{H}}(\xi)=\left\langle\mathbf{X} ; T_{H}\right\rangle_{\xi}$, allowing the use of a smoothed interpolation [39] without sacrificing accuracy in the projected free energy gradient. We stress that the change of candidate pathway at high temperature is to satisfy the locality condition (6), not to enhance sampling at low temperature as in replica exchange strategies [9]. While a detailed exposition of adaptively updating the candidate pathway will be the topic of a future publication, a central advantage of the method presented here is that for a large class of solid state systems this complication does not arise; the MEP locality condition (6) is satisfied when the convexity of the energy landscape is robust under a finite temperature. In the vast majority of solid state systems, the convexity of the energy landscape is determined by the local structural environment around each atom, which in turn is typically robust below the melting temperature aside from the transition of interest. Importantly, if the convexity of the energy landscape changes sufficiently to violate (6), our method will clearly indicate that a converged result cannot be found, and the concept of a free energy difference between the proposed end states becomes ill defined. In general, for systems where a converged minimum energy pathway can be found, our main result (8) can typically be used to extract the exact free energy difference below the melting temperature. Using a modified version of the LAMMPS simulation package [40], an initial pathway was sampled using overdamped Brownian dynamics, integrating the stochastic equation of motion

$$
\gamma \dot{\mathbf{X}}(t)=-\mathbf{Q} \cdot \boldsymbol{\nabla} V[\mathbf{X}(t)]+\sqrt{2 \gamma k_{B} T} \mathbf{Q} \cdot \boldsymbol{\eta}(t),
$$

where $\gamma$ is a friction coefficient chosen for numerical stability, $\boldsymbol{\eta}(t) \in \mathbb{R}^{3 N}$ is white noise with autocorrelation $\left\langle\boldsymbol{\eta}(t) \otimes \boldsymbol{\eta}\left(t^{\prime}\right)\right\rangle=\delta\left(t-t^{\prime}\right) \rrbracket$, and $\mathbf{Q} \equiv \mathbb{\square} \hat{\mathbf{n}} \otimes \hat{\mathbf{n}} \in \mathbb{R}^{3 N \times 3 N}$ keeps the system on a hyperplane defined by the unit normal vector $\hat{\mathbf{n}}=\mathbf{n} /|\mathbf{n}|$, with additional constraints used to stop the center of mass drift through a Gram-Schmidt process. The initial reaction pathway $\mathbf{X}_{0}(\xi)$ is produced by a spline interpolation of images from a NEB calculation. An ensemble of typically 100-1000 independent trajectories are generated in parallel according to (9), with $\mathbf{n}=\partial_{\xi} \mathbf{X}_{0}(\xi)$ for initially 10 values of $\xi$ in $[0,1]$; though if the calculated mean force was large, additional samples at intermediate $\xi$ values were taken. We note that this quadrature issue is readily automated. Each system trajectory consists of a thermalization stage of 1000-4000 integration steps, with the system temperature monitored through the equipartition relation $k_{B} T=\langle V(\mathbf{X})-$ $\left.V\left(\mathbf{X}_{0}\right)\right\rangle / 1.5 N$ and the simulation box rescaled to account for thermal expansion. Once thermalized, the free energy gradient (8) is recorded along with the path deviation $\delta \mathbf{X}(\xi)=\mathbf{X}_{\mathrm{T}}(\xi)-\mathbf{X}_{0}(\xi)$ over trajectories of typically 2000-5000 steps, producing a calculation of the free energy gradient and the minimum free energy path. We have verified that the sampling error of both quantities scales as the inverse square root of the number of force samples and is largely independent of system size, showing ideal sampling statistics. An ensemble size of $100-500$ 
independent simulations typically gave an acceptable sampling error in (8).

Application to interstitial cluster transformation $\alpha$ iron.-The first application is a complex transformation pathway in $\alpha$ iron that links disjoint basins of $C 15$ clusters [41] and traditional clusters of $\langle 110\rangle$ dumbbells [42]. The transformation of $C 15$ clusters to $\langle 110\rangle$ dumbbell clusters are of great interest in studies of radiation damage as they have been postulated to play a key role in controlling the relative population of $\langle 111\rangle$ and $\langle 100\rangle$ interstitial loops [43-46].

The initial pathway was prepared using the activation relaxation technique $[19,22,23,41]$ to find the pathway saddle point, before running a NEB calculation to find the minimum energy pathway. The full transformation pathway is complex and nonintuitive, involving the coordinated displacements of multiple atoms, for which designing a set of collective variable functions is either impossible or impractically challenging. In the current approach, we simply use the MEP $\mathbf{X}_{0}(\xi)$ to determine the reaction coordinate as described above, using an ensemble of 1000 independent simulations to produce a free energy profile at 10 temperatures up to $900 \mathrm{~K}$.

The results of our calculation are shown in Fig. 1, where we also plot the harmonic free energy difference computed from diagonalization of Hessian matrices along the path [6]. While it can be seen that the true free energy barrier $\Delta \mathcal{F}=\max \mathcal{F}(\xi)-\mathcal{F}(0)$ and total free energy difference $\mathcal{F}(1)-\mathcal{F}(0)$ agree with the harmonic approximation below $200 \mathrm{~K}$, above this modest temperature a strong anharmonic component is seen in both cases. In particular,

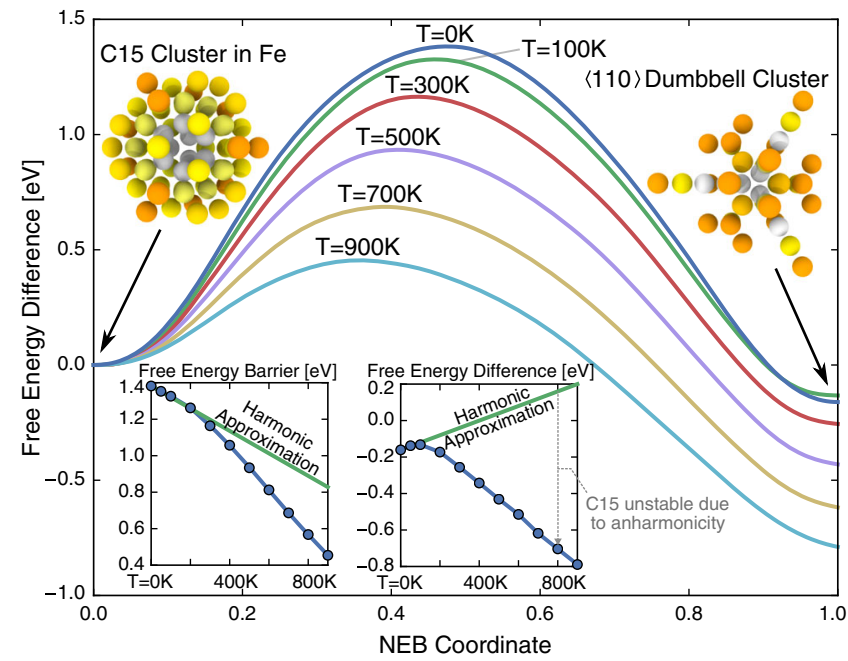

FIG. 1. Free energy profile for the transformation of a $C 15$ interstitial cluster to a cluster of $\langle 110\rangle$ dumbbell interstitials in an EAM of $\alpha$ iron [41]. Insets: Free energy barrier and total free energy difference for the transformation. The harmonic free energy $\mathcal{F}_{0}$, obtained by diagonalizing the Hessian matrix, is only valid up to around $200 \mathrm{~K}$. For an ensemble size of more than 300 , we find the ensemble error is not visible on the scale shown. from the population ratio $n_{1} / n_{0}$, we see that the harmonic approximation predicts that the $C 15$ cluster has relative stability to the $\langle 110\rangle$ dumbbell clusters above $450 \mathrm{~K}$, while the full anharmonic calculation completely reverses this picture, transforming the expected population of $C 15$ clusters under experimentally relevant conditions. These unsupervised simulations open the possibility of investigation of the complex transformation of multiple interstitials clusters and can answer important questions related to the formation of $C 15$ in the regime of room temperature, observed by Arakawa [47], as well as the well-known open question of the interstitial loop character at high temperature [43]. Finally, access to the free energy migration barriers for general defects significantly improves agreement with resistivity recovery experiments of irradiated materials $[48,49]$, for which a detailed study is outside the scope of this Letter and will be presented elsewhere.

Application to double kink nucleation on a $\langle 111\rangle$ screw dislocation in tungsten.-The dislocation plasticity of bcc metals is known to be controlled in large part by the kinklimited motion of $\langle 111\rangle$ screw dislocations [1,50-53], with kink nucleation considered to be the central mechanism of bcc plasticity [54]. Direct molecular dynamics studies of the kink nucleation process must apply unrealistically large stresses of $O(\mathrm{GPa})$ to generate sufficient dislocation motion within the few nanoseconds of trajectory; however, this is known to significantly affect the nature of the migration potential [55] and has an unclear relationship with the high temperature, $O(10 \mathrm{MPa})$ stress regime of experimental relevance. The harmonic free energy barrier for screw dislocation motion has recently been evaluated for $\alpha$ iron [1], and a free energy calculation for a short, straight, screw dislocation segment has been evaluated using the Bennet acceptance ratio method, with the goal to extract the Peierls free energy barrier [56] for rigid dislocation motion.

We have exploited the efficiencies of the method to evaluate the free energy barrier for the full double kink nucleation process in an embedded atom model (EAM) of tungsten [57]. As illustrated in Fig. 2, the system has an initially straight screw dislocation lying along [111] in a monoclinic simulation cell with bounding (101) planes [58]. The glide process proceeds along [121], with initial NEB calculations giving a double kink formation energy of $1.67 \mathrm{eV}$. As the total system contains around 120000 atoms, these calculations represent a genuine advance in the size of systems which can be treated with free energy calculation methods. As in the previous example, it can be seen that the harmonic approximation breaks down by $200 \mathrm{~K}$, with the sign of the entropy changing. This anharmonicity has a significant effect on the expected double kink velocity experimentally relevant temperatures, which is approximately proportional to $\exp (-\beta \Delta \mathcal{F})$ at low applied stresses [7]. In Fig. 2, we plot the ratio of expected screw dislocation velocities using the harmonic and 


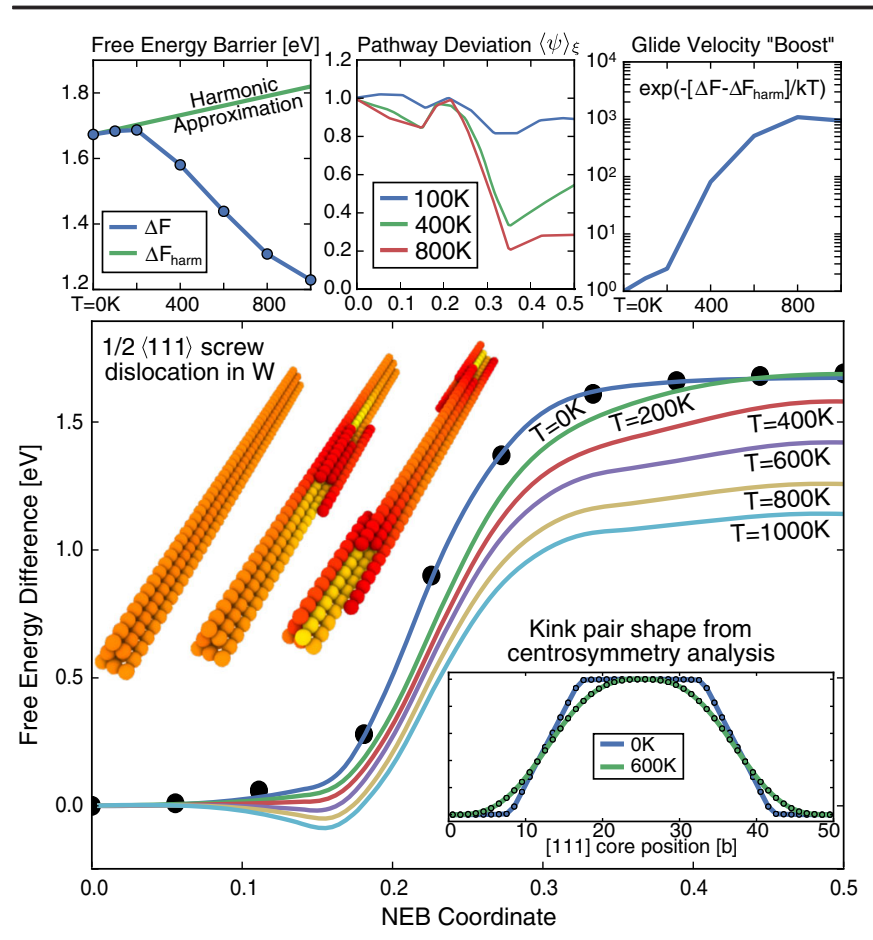

FIG. 2. Free energy for double kink nucleation for a $\langle 111\rangle$ screw in $W$. Inset: Kink profile determined through centrosymmetry analysis on the time averaged atomic positions [51]. Top left: Kink formation free energy. Top center: Pathway deviation $\langle\psi\rangle_{\xi}$. Top right: Effective "boost" in the expected glide velocity due to anharmonic corrections.

anharmonic double kink formation free energies, namely, $\exp \left(-\beta\left[\Delta \mathcal{F}-\Delta \mathcal{F}_{\text {harm }}\right]\right)$, where it can be seen that the anharmonic reduction in $\Delta F$ causes the predicted velocity to be over $\times 10^{3}$ greater at $800 \mathrm{~K}$. These results already demonstrate that anharmonic effects must be considered even at low homologous temperatures to correctly capture important plasticity processes, including understanding the flow regimes of bcc metals [7,59]. We note that the anharmonic corrections to the kink formation free energy increase from around $200 \mathrm{~K}$, where experiments have shown a clear change in the flow stress temperature dependence [59]; the extension of our method to stress controlled simulations will greatly aid in this investigation and is a topic for future study.

Conclusion.-This Letter presents a parametrization-free path-based method to calculate anharmonic free energy differences of thermally activated mechanisms in the solid state. By removing the need to define ad hoc collective variable functions, our method allows the automated investigation of anharmonicity in arbitrarily complex multiatom diffusion processes that were previously impossible or impractical to evaluate. The method was demonstrated on two systems of great importance, where the clear influence of anharmonicity was shown to be present even at low homologous temperatures. The possible applications to materials science are very broad; future work will focus on integrating these methods into automated simulation schemes [25], exploiting the optimality [9] of our approach in $a b$ initio sampling strategies [60], and incorporating nonequilibrium boundary conditions, in particular, the application of external stresses.

The authors acknowledge insightful discussions with T. Lelièvre and G. Stoltz. This work was carried out within the EUROfusion Consortium with support from the EURATOM research and training program 2014-2018 Grant No. 633053, GENCI-(CINES/CCRT) Grant No. A0030906973, PRACE/Marconi CINECA (MORPHO project) and RCUK Energy Program Grant No. EP/P012450/1.

*Present address: Theoretical Division T-1, Los Alamos National Laboratory, Los Alamos, New Mexico, 87545, USA.

tomswinburne@gmail.com mihai-cosmin.marinica@cea.fr

[1] L. Proville, D. Rodney, and M. C. Marinica, Nat. Mater. 11, 845 (2012).

[2] S. M. Keralavarma, T. Cagin, A. Arsenlis, and A. A. Benzerga, Phys. Rev. Lett. 109, 265504 (2012).

[3] T. D. Swinburne, K. Arakawa, H. Mori, H. Yasuda, M. Isshiki, K. Mimura, M. Uchikoshi, and S. L. Dudarev, Sci. Rep. 6, 30596 (2016).

[4] D. Caillard and J.L. Martin, Thermally Activated Mechanisms in Crystal Plasticity, Pergamon Materials Series (Elsevier Science, New York, 2003).

[5] D. J. Wales, Energy Landscapes (Cambridge University Press, Cambridge, England, 2004).

[6] P. Hänggi, P. Talkner, and M. Borkovec, Rev. Mod. Phys. 62, 251 (1990).

[7] D. Cereceda, M. Diehl, F. Roters, D. Raabe, J. M. Perlado, and J. Marian, Int. J. Plast. 78, 242 (2016).

[8] G. Henkelman, Annu. Rev. Mater. Res. 47, 199 (2017).

[9] T. Lelièvre, G. Stoltz, and M. Rousset, Free Energy Computations: A Mathematical Perspective (Imperial College Press, London, 2010).

[10] G. M. Torrie and J. P. Valleau, J. Comput. Phys. 23, 187 (1977).

[11] S. Kumar, J. M. Rosenberg, D. Bouzida, R. H. Swendsen, and P. A. Kollman, J. Comput. Chem. 13, 1011 (1992).

[12] E. Darve, D. Rodríguez-Gómez, and A. Pohorille, J. Chem. Phys. 128, 144120 (2008).

[13] A. F. Voter, Phys. Rev. Lett. 78, 3908 (1997).

[14] A. Laio and M. Parrinello, Proc. Natl. Acad. Sci. U.S.A. 99, 12562 (2002).

[15] W. E, W. Ren, and E. Vanden-Eijnden, Phys. Rev. B 66, 052301 (2002).

[16] E. Weinan, W. Ren, and E. Vanden-Eijnden, J. Phys. Chem. B 109, 6688 (2005).

[17] A. K. Faradjian and R. Elber, J. Chem. Phys. 120, 10880 (2004).

[18] D. Branduardi, F. L. Gervasio, and M. Parrinello, J. Chem. Phys. 126, 054103 (2007). 
[19] M.-C. Marinica, F. Willaime, and N. Mousseau, Phys. Rev. B 83, 094119 (2011).

[20] L. Cao, G. Stoltz, T. Lelièvre, M.-C. Marinica, and M. Athènes, J. Chem. Phys. 140, 104108 (2014).

[21] J. Duncan, A. Harjunmaa, R. Terrell, R. Drautz, G. Henkelman, and J. Rogal, Phys. Rev. Lett. 116, 035701 (2016).

[22] F. El-Mellouhi, N. Mousseau, and L. J. Lewis, Phys. Rev. B 78, 153202 (2008).

[23] L. K. Béland, P. Brommer, F. El-Mellouhi, J.-F. Joly, and N. Mousseau, Phys. Rev. E 84, 046704 (2011).

[24] D. Perez, B. P. Uberuaga, Y. Shim, J. G. Amar, and A. F. Voter, Annu. Rep. Comput. Chem. 5, 79 (2009).

[25] R. J. Zamora, B. P. Uberuaga, D. Perez, and A. F. Voter, Annu. Rev. Chem. Biomol. Eng. 7, 87 (2016).

[26] L. M. Ghiringhelli, J. Vybiral, S. V. Levchenko, C. Draxl, and M. Scheffler, Phys. Rev. Lett. 114, 105503 (2015).

[27] A. Jain, S. P. Ong, G. Hautier, W. Chen, W. D. Richards, S. Dacek, S. Cholia, D. Gunter, D. Skinner, G. Ceder et al., APL Mater. 1, 011002 (2013).

[28] Z. Li, J. R. Kermode, and A. De Vita, Phys. Rev. Lett. 114, 096405 (2015).

[29] E. Darve, D. Rodríguez-Gómez, and A. Pohorille, J. Chem. Phys. 128, 144120 (2008).

[30] C. Dellago, P. Bolhuis, and P. L. Geissler, Adv. Chem. Phys. 123 (2002).

[31] L. Maragliano, A. Fischer, E. Vanden-Eijnden, and G. Ciccotti, J. Chem. Phys. 125, 024106 (2006).

[32] L. Maragliano and E. Vanden-Eijnden, Chem. Phys. Lett. 446, 182 (2007).

[33] E. Vanden-Eijnden and M. Venturoli, J. Chem. Phys. 131, 044120 (2009).

[34] L. D. Nguyen, K. L. Baker, and D. H. Warner, Phys. Rev. B 84, 024118 (2011).

[35] S. Saroukhani and D. Warner, Acta Mater. 128, 77 (2017).

[36] C. Huang, A. F. Voter, and D. Perez, Phys. Rev. B 87, 214106 (2013).

[37] G. Henkelman, B. P. Uberuaga, and H. Jonsson, J. Chem. Phys. 113, 9901 (2000).

[38] C. Chipot and A. Pohorille, Free Energy Calculations (Springer, New York, 2007).

[39] G. Wahba, Spline Models for Observational Data (Society for Industrial and Applied Mathematics, Philadelphia, 1990).
[40] S. Plimpton, J. Comput. Phys. 117, 1 (1995).

[41] M.-C. Marinica, F. Willaime, and J.-P. Crocombette, Phys. Rev. Lett. 108, 025501 (2012).

[42] D. A. Terentyev, T. P. C. Klaver, P. Olsson, M.-C. Marinica, F. Willaime, C. Domain, and L. Malerba, Phys. Rev. Lett. 100, 145503 (2008).

[43] S. L. Dudarev, R. Bullough, and P. M. Derlet, Phys. Rev. Lett. 100, 135503 (2008).

[44] L. Dézerald, M.-C. Marinica, L. Ventelon, D. Rodney, and F. Willaime, J. Nucl. Mater. 449, 219 (2014).

[45] Y. Zhang, X.-M. Bai, M. R. Tonks, and S. B. Biner, Scr. Mater. 98, 5 (2015).

[46] R. Alexander, M.-C. Marinica, L. Proville, F. Willaime, K. Arakawa, M. R. Gilbert, and S. L. Dudarev, Phys. Rev. B 94, 024103 (2016).

[47] K. Arakawa (to be published).

[48] M. Kiritani, H. Takata, K. Moriyama, and F. Fujita, Philos. Mag. A 40, 779 (1979).

[49] C.-C. Fu, J. Dalla Torre, F. Willaime, J.-L. Bocquet, and A. Barbu, Nat. Mater. 4, 68 (2005).

[50] S. Queyreau, J. Marian, M. R. Gilbert, and B. D. Wirth, Phys. Rev. B 84, 064106 (2011).

[51] T. D. Swinburne, S. L. Dudarev, S. P. Fitzgerald, M. R. Gilbert, and A.P. Sutton, Phys. Rev. B 87, 064108 (2013).

[52] L. Dezerald, L. Ventelon, E. Clouet, C. Denoual, D. Rodney, and F. Willaime, Phys. Rev. B 89, 024104 (2014).

[53] L. Dezerald, D. Rodney, E. Clouet, L. Ventelon, and F. Willaime, Nat. Commun. 7, 11695 (2016).

[54] J. P. Hirth and J. Lothe, Theory of Dislocations (Krieger Publishing Company, Malabar, FL, 1991).

[55] D. Rodney and L. Proville, Phys. Rev. B 79, 094108 (2009).

[56] M. R. Gilbert, P. Schuck, B. Sadigh, and J. Marian, Phys. Rev. Lett. 111, 095502 (2013).

[57] M. C. Marinica, L. Ventelon, M. R. Gilbert, L. Proville, S. L. Dudarev, J. Marian, G. Bencteux, and F. Willaime, J. Phys. Condens. Matter 25, 395502 (2013).

[58] J. Chaussidon, M. Fivel, and D. Rodney, Acta Mater. 54, 3407 (2006).

[59] D. Brunner, Mater. Trans., JIM 41, 152 (2000).

[60] B. Grabowski, L. Ismer, T. Hickel, and J. Neugebauer, Phys. Rev. B 79, 134106 (2009). 